\title{
INTEGRAL CLOSURE OF A GRADED NOETHERIAN DOMAIN
}

\author{
Chang Hwan Park and Mi Hee Park
}

\begin{abstract}
We show that, if $R$ is a graded Noetherian ring and $I$ is a proper ideal of $R$ generated by $n$ homogeneous elements, then any prime ideal of $R$ minimal over $I$ has h-height $\leq n$, and that if $R$ is a graded Noetherian domain with h-dim $R \leq 2$, then the integral closure $R^{\prime}$ of $R$ is also a graded Noetherian domain with h-dim $R^{\prime} \leq 2$. We also present a short improved proof of the result that, if $R$ is a graded Noetherian domain, then the integral closure of $R$ is a graded Krull domain.
\end{abstract}

\section{Introduction}

Let $\Gamma$ be a torsion-free cancellative commutative monoid with quotient group $G$ and let $R=\bigoplus_{\gamma \in \Gamma} R_{\gamma}$ be a $\Gamma$-graded commutative ring. A goal of this paper is to generalize many of the well known results for Noetherian rings to graded Noetherian rings.

A $\Gamma$-graded commutative ring $R=\bigoplus_{\gamma \in \Gamma} R_{\gamma}$ is said to be graded Noetherian if $R$ satisfies the ascending chain condition (a.c.c.) on homogeneous ideals, or equivalently, if each homogeneous ideal of $R$ is finitely generated.

Goto and Yamagishi [7, Theorem 1.1] show that, if $\Gamma$ is a finitely generated abelian group, then $R=\bigoplus_{\gamma \in \Gamma} R_{\gamma}$ is graded Noetherian if and only if it is Noetherian. One of the most natural and important examples of a $\Gamma$-graded ring is the monoid ring $R[\Gamma]=\bigoplus_{\gamma \in \Gamma} R X^{\gamma}$. It is shown in [18, Theorem 2.4] that a $\Gamma$-monoid ring $R[\Gamma]$ is graded Noetherian if and only if $R$ is Noetherian and each ideal of $\Gamma$ is finitely generated. In view of a result of Budach [3] (see [18, Theorem 2.2]), it follows that $R[\Gamma]$ is Noetherian if and only if $R$ is Noetherian and $\Gamma$ is finitely generated.

Thus if $k$ is a field and $G$ is an abelian group which is not finitely generated, then the $G$-group ring $k[G]$ is an example of a graded Noetherian ring which is not Noetherian.

Received November 12, 2009; Revised June 10, 2010.

2010 Mathematics Subject Classification. 13A02, 13A15, 13B22, 13E05, $13 \mathrm{~F} 05$.

Key words and phrases. graded ring, graded module, Noetherian ring, Krull domain, integral closure.

This work was supported by the Korea Research Foundation Grant funded by the Korean Government (MOEHRD) (KRF-2006-531-C00006). 
After giving some needed definitions and preliminary results in Section 2, we show in Section 3 that, if $R$ is a graded Noetherian ring and $I$ is a proper ideal of $R$ generated by $n$ homogeneous elements, then any prime ideal of $R$ minimal over $I$ has h-height $\leq n$ (Theorem 3.6).

In Section 4 we prove that, if $R$ is a $\Gamma$-graded Noetherian domain with h-dim $R=1$, then any homogeneous overring of $R$ is $G$-graded Noetherian with h-dimension $\leq 1$. In fact, a more general statement is proved: Let $R \subseteq A$ be graded integral domains with homogeneous quotient fields $K \subseteq L$, respectively. If $R$ is graded Noetherian with h-dim $R=1$ and $L$ is finite over $K$, then $A$ is graded Noetherian with h-dim $A \leq 1$ (Theorem 4.2).

In Section 5, as an application of Theorem 3.6 and Theorem 4.2, we give an alternate proof of [16, Theorem 2.10] or [15, Theorem 9.1]: If $R$ is a $\Gamma$-graded Noetherian domain, then the integral closure of $R$ is a $G$-graded Krull domain (Theorem 5.3).

In Section 6 we show that, if $R$ is a graded Noetherian domain with h-dim $R \leq$ 2 , then the integral closure $R^{\prime}$ of $R$ is also a graded Noetherian domain with h- $\operatorname{dim} R^{\prime} \leq 2$ (Theorem 6.8).

In the last section, we recall some well known results whose graded versions fail to hold.

If the grading by $\Gamma$ is trivial, i.e., every element of the graded ring $R$ has degree zero, then Theorem 3.6, Theorem 4.2, Theorem 5.3, and Theorem 6.8 are exactly the same as the generalized principal ideal theorem of Krull [11, Theorem 152], the Krull-Akizuki theorem [12, Theorem 11.7], the Mori-Nagata theorem [13, Theorem 33.10], and the Nagata theorem [13, Theorem 33.12], respectively.

As mentioned earlier, there exist examples of graded Noetherian rings which are not Noetherian. Thus the graded statements given in this paper could be considered to be real generalizations of the corresponding non-graded statements.

For more information on graded rings, the readers are referred to $[1,5,6,7$, $9,15,16,17,18]$.

\section{Preliminaries}

This section contains several definitions and a few known results that will be used in what follows.

Throughout the paper, we let $\Gamma$ be a torsion-free cancellative commutative monoid with quotient group $G$ and let $R=\bigoplus_{\gamma \in \Gamma} R_{\gamma}$ be a $\Gamma$-graded commutative ring with identity, unless otherwise stated.

Definition 2.1. Let $R$ be a graded ring.

(1) Let h-Spec $(R)$ denote the set of homogeneous prime ideals of $R$ and let h-Max $(R)$ denote the set of ideals of $R$ which are maximal in the set of all proper homogeneous ideals of $R$. Then h-Max $(R) \subseteq \mathrm{h}-\operatorname{Spec}(R)$. 
(2) The h-height of a homogeneous prime ideal $P$ (denoted by h-ht $P$ ) is defined to be the supremum of the lengths of chains of homogeneous prime ideals descending from $P$.

(3) The h-dimension of $R$ (denoted by h-dim $R$ ) is defined to be $\sup \{\mathrm{h}-\mathrm{ht} P \mid$ $P \in \mathrm{h}-\operatorname{Spec}(R)\}$.

(4) $R$ is called a graded Noetherian ring if $R$ satisfies the ascending chain condition (a.c.c.) on homogeneous ideals.

Definition 2.2. Let $R$ be a graded integral domain.

(1) Let $S$ be the set of nonzero homogeneous elements of $R$, then $S$ is a multiplicatively closed set. The quotient ring $R_{S}$ is a $G$-graded ring, where $R_{S}=\bigoplus_{\gamma \in G}\left(R_{S}\right)_{\gamma}$ with each $\left(R_{S}\right)_{\gamma}=\left\{\frac{a}{b} \mid a \in R_{\alpha}, b \in R_{\beta} \backslash\right.$ $\{0\}$, and $\alpha-\beta=\gamma\}$, and it is called the homogeneous quotient field of $R$. Note that each nonzero homogeneous element of $R_{S}$ is a unit.

(2) An overring $A$, with $R \subseteq A \subseteq R_{S}$, is called a homogeneous overring if $A=\bigoplus_{\gamma \in G}\left(A \cap\left(R_{S}\right)_{\gamma}\right)$. Thus $A$ is a $G$-graded integral domain with $A_{\gamma}=A \cap\left(R_{S}\right)_{\gamma}$ for each $\gamma \in G$.

(3) The h-global transform of $R$ is defined to be the set $R^{h g}=\left\{x \in R_{S} \mid\right.$ $M_{1} \cdots M_{k} x \subseteq R$, where $M_{i} \in \mathrm{h}-\operatorname{Max}(R)$, not necessarily distinct, $k \geq$ 1 . Note that $R^{h g}$ is a homogeneous overring of $R$.

(4) Let $\left\{R_{\lambda}\right\}_{\lambda \in \Lambda}$ be a family of homogeneous overrings of $R$ such that $R=\bigcap R_{\lambda}$. The intersection $R=\bigcap R_{\lambda}$ is said to be homogeneously locally finite if each nonzero homogeneous element of $R$ is a unit in all $R_{\lambda}$ but a finite number of the $R_{\lambda}$.

(5) $R$ is called a graded DVR if $R$ has the unique nonzero homogeneous prime ideal and it is principal (generated by a homogeneous element).

(6) $R$ is called a graded Krull domain if it is completely integrally closed and satisfies the a.c.c. on homogeneous $v$-ideals (For the definition of $v$-ideal, see Section 5).

Theorem 2.3. Let $R$ be a graded ring.

(1) [18, Lemma 2.3] $R$ is a graded Noetherian ring if and only if each homogeneous prime ideal of $R$ is finitely generated.

(2) [1, Theorem 5.15] Assume, moreover, that $R$ is an integral domain. Then $R$ is a graded Krull domain if and only if $R=\bigcap V_{\lambda}$, where the intersection is homogeneously locally finite and each $V_{\lambda}$ is a homogeneous overring of $R$ which is a graded DVR.

Theorem 2.4. Let $R$ be a graded Noetherian domain.

(1) $\left[16\right.$, Lemma 2.2 and Lemma 2.3] The complete integral closure $R^{*}$ and the integral closure $R^{\prime}$ of $R$ are the same and it is a homogeneous overring of $R$.

(2) [16, Theorem 1.4] Let $A$ be a homogeneous overring of $R$ contained in $R^{\text {hg }}$. Then $A$ is a graded Noetherian domain. 


\section{The principal ideal theorem}

In this section we prove a graded version of the principal ideal theorem of Krull. The proof we give is adapted from [11].

We say that a nonzero graded $R$-module $M$ is $h$-irreducible if $M$ has no nontrivial homogeneous submodules. Note that any h-irreducible graded $R$ module is isomorphic to $R / m$ as graded $R$-modules, where $m$ is a maximal homogeneous ideal of $R$.

For a nonzero graded $R$-module $M$, a chain $M=M_{0} \supset M_{1} \supset \cdots \supset M_{r}=$ (0) of homogeneous submodules of $M$ is called an h-composition series of $M$ if each $M_{i} / M_{i+1}$ is h-irreducible; the integer $r$ is called the length of the hcomposition series.

Theorem 3.1 (cf. Jordan-Hölder [19, Chapter III, §11, Theorem 19]). If a nonzero graded module $M$ has an h-composition series of length $r$, then any $h$ composition series of $M$ has length $r$ and any chain of homogeneous submodules of $M$ can be refined to an h-composition series.

Proof. We prove the theorem by induction on $r$. If $r=1$, then the assertion is obvious. Let $r>1$. Assume that the theorem is true for graded modules having an h-composition series of length less than $r$.

Let $M=M_{0} \supset M_{1} \supset \cdots \supset M_{r}=(0)$ be an h-composition series of length $r$. Then by the induction hypothesis $M$ can have no h-composition series of length less than $r$. It suffices to show that any chain of homogeneous submodules of $M$ has length at most $r$.

Let $M=N_{0} \supset N_{1} \supset \cdots \supset N_{s}=(0)$ be a chain of distinct homogeneous submodules of $M$. If $N_{1}=M_{1}$, then since $M_{1}$ has an h-composition series $M_{1} \supset \cdots \supset M_{r}=(0)$ of length $r-1$, by the induction hypothesis $s-1 \leq r-1$ and hence $s \leq r$. If $N_{1} \subset M_{1}$, then $M_{1} \supset N_{1} \supset N_{2} \supset \cdots \supset N_{s}=(0)$ is a chain of homogeneous submodules of $M_{1}$ of length $s$. Again by the induction hypothesis, $s \leq r-1$ and so $s \leq r$.

Assume that $N_{1} \nsubseteq M_{1}$. Then $M_{1}+N_{1}=M$. Note that $M / M_{1}=\left(M_{1}+\right.$ $\left.N_{1}\right) / M_{1} \cong N_{1} /\left(M_{1} \cap N_{1}\right)$ as graded modules. Since $M / M_{1}$ is h-irreducible, so is $N_{1} /\left(M_{1} \cap N_{1}\right)$.

Since $M_{1}$ has an h-composition series of length $r-1$ and $M_{1} \supset M_{1} \cap N_{1}$, by the induction hypothesis $M_{1} \cap N_{1}$ has an h-composition series of length at most $r-2$. Then since there are no homogeneous submodules between $N_{1}$ and $M_{1} \cap N_{1}, N_{1}$ has an h-composition series of length at most $r-1$. By the induction hypothesis, $s-1 \leq r-1$ and hence $s \leq r$.

The length of an h-composition series of $M$ is called the h-length of $M$ and denoted by hl $M$.

Lemma 3.2. Let $R$ be a graded integral domain with $\mathrm{h}-\operatorname{dim} R=0$. Then every finitely generated graded $R$-module has finite h-length. 
Proof. Note that, since $R$ has no nontrivial homogeneous ideals, $R$ as a graded $R$-module is h-irreducible. Let $A$ be a nonzero finitely generated graded $R$ module. Write $A=R x_{1}+\cdots+R x_{n}$, where each $x_{i}$ is a homogeneous element of $A$. We will use induction on $n$. If $n=1$, then $A=R x_{1} \cong R /\left(0:_{R} x_{1}\right)$ as graded $R$-modules. Since $R$ is h-irreducible, $\left(0:_{R} x_{1}\right)=(0)$ and $A$ is h-irreducible, i.e., hl $A=1$. Let $n>1$ and set $B:=R x_{2}+\cdots+R x_{n}$. Then by the induction hypothesis, $B$ has finite h-length. Consider the chain $A \supseteq B \supseteq(0)$. Since $A / B \cong R \bar{x}_{1}$, it is $(0)$ or h-irreducible. Therefore, hl $A=\operatorname{hl}(A / B)+\mathrm{hl} B \leq$ $1+$ hl $B<\infty$.

Lemma 3.3. Let $R$ be a graded Noetherian ring with $\mathrm{h}-\operatorname{dim} R=0$. Then every finitely generated graded $R$-module has finite h-length.

Proof. Since h-dim $R=0, \operatorname{Min}(R)=\mathrm{h}-\operatorname{Spec}(R)=\mathrm{h}-\operatorname{Max}(R)$. By [16, Corollary 1.2], there are only a finite number of minimal prime ideals, say $P_{1}, \ldots, P_{n}$. Then we have $P_{1} \cdots P_{n} \subseteq P_{1} \cap \cdots \cap P_{n}=\sqrt{(0)}$. Since $\sqrt{(0)}$ is a homogeneous ideal, it is finitely generated and hence $\sqrt{(0)}^{k}=(0)$ for some $k$, which implies that $\left(P_{1} \cdots P_{n}\right)^{k}=(0)$.

Let $A$ be a nonzero finitely generated graded $R$-module, and consider the chain of homogeneous submodules

$$
\begin{aligned}
A & \supseteq P_{1} A \supseteq P_{1}^{2} A \supseteq \cdots \supseteq P_{1}^{k} A \supseteq P_{1}^{k} P_{2} A \supseteq P_{1}^{k} P_{2}^{2} A \\
& \supseteq \cdots \supseteq P_{1}^{k} P_{2}^{k} A \supseteq P_{1}^{k} P_{2}^{k} P_{3} A \supseteq \cdots \supseteq P_{1}^{k} P_{2}^{k} \cdots P_{n}^{k} A=(0) .
\end{aligned}
$$

Let $B$ and $P_{i} B$ be any two consecutive modules in this chain. Then since each $P_{j}$ is a finitely generated homogeneous ideal of $R$ and $A$ is a finitely generated graded $R$-module, $B / P_{i} B$ is a finitely generated graded module over $R / P_{i}$. Since $R / P_{i}$ is a graded integral domain with h-dim $R / P_{i}=0, B / P_{i} B$ has finite h-length by Lemma 3.2. Therefore, hl $\left(B / P_{i} B\right)<\infty$, and the sum hl $A$ of these terms is also finite.

Lemma 3.4. Let $u, y$ be nonzero homogeneous elements in a graded integral domain. Then

(1) The modules $(u, y) /(u)$ and $\left(u^{2}, u y\right) /\left(u^{2}\right)$ are isomorphic as graded modules.

(2) Assume further that $t u^{2} \in(y)$ implies $t u \in(y)$. Then the modules $(u) /\left(u^{2}\right)$ and $\left(u^{2}, y\right) /\left(u^{2}, u y\right)$ are isomorphic as graded modules.

Proof. See the proof of [11, Theorem 143].

(1) Since $u$ is a homogeneous element, the multiplication by $u$ induces a graded module isomorphism of $(u, y) /(u)$ onto $\left(u^{2}, u y\right) /\left(u^{2}\right)$.

(2) Multiplication by $u$ induces a graded module isomorphism of $R /(u)$ onto $(u) /\left(u^{2}\right)$. Also, under the given assumption, multiplication by $y$ induces a graded module isomorphism of $R /(u)$ onto $\left(u^{2}, y\right) /\left(u^{2}, u y\right)$. 
Theorem 3.5 (cf. Principal Ideal Theorem [11, Theorem 142]). Let $x$ be a nonunit homogeneous element in a graded Noetherian ring $R$ and let $P$ be a prime ideal minimal over $(x)$. Then h-ht $P \leq 1$.

Proof. Suppose on the contrary that there is a chain $P \supset P_{1} \supset P_{2}$ of distinct homogeneous prime ideals. Replacing $R$ by $R / P_{2}$, and then considering $R_{S \backslash P}$, where $S$ is the set of nonzero homogeneous elements of $R$, we may assume that $R$ is a graded Noetherian domain with the unique maximal homogeneous ideal $P$.

Choose a nonzero homogeneous element $y$ in $P_{1}$. Let $I_{k}$ denote the ideal $\left((y):_{R} x^{k}\right), k=1,2, \ldots$ Then $I_{1} \subseteq I_{2} \subseteq \ldots$ is an ascending chain of homogeneous ideals. Since $R$ is graded Noetherian, the chain must become stable, say at $I_{n}$. Then $t \in\left((y): x^{2 n}\right)$ implies $t \in\left((y): x^{n}\right)$. Set $u=x^{n}$, then $t u^{2} \in(y)$ implies $t u \in(y)$. The ring $T=R /\left(u^{2}\right)$ is a graded Noetherian ring with exactly one homogeneous prime ideal, and hence h-dim $T=0$. Therefore, by Lemma 3.3, every finitely generated graded $T$-module has finite h-length. This applies in particular to the module $(u, y) /\left(u^{2}\right)$, which is a graded $R$-module annihilated by $u^{2}$ and hence a graded $T$-module. It follows from Lemma 3.4 that $\mathrm{hl}\left((u, y) /\left(u^{2}\right)\right)=\mathrm{hl}((u, y) /(u))+\mathrm{hl}\left((u) /\left(u^{2}\right)\right)=\mathrm{hl}\left(\left(u^{2}, u y\right) /\left(u^{2}\right)\right)+$ hl $\left(\left(u^{2}, y\right) /\left(u^{2}, u y\right)\right)=\mathrm{hl}\left(\left(u^{2}, y\right) /\left(u^{2}\right)\right)$. Thus the graded $T$-module $(u, y) /\left(u^{2}\right)$ and its graded submodule $\left(u^{2}, y\right) /\left(u^{2}\right)$ have the same h-length. This is possible only if $(u, y)=\left(u^{2}, y\right)$, i.e., $u \in\left(u^{2}, y\right)$. Write $u=c u^{2}+d y$, where $c, d$ are homogeneous elements in $R$. Then $1-c u \in\left((d y):_{R} u\right)$. Since $P$ is the unique maximal homogeneous ideal of $R$ and $1-c u \notin P$, the homogeneous ideal $\left((d y):_{R} u\right)$ must be equal to $R$. Thus we have $u \in(d y) \subseteq(y) \subseteq P_{1}$. But $P$ is minimal over $(x)$ and hence also minimal over $(u)=\left(x^{n}\right)$. This contradiction completes the proof.

Theorem 3.6 (cf. Generalized Principal Ideal Theorem [11, Theorem 152]). Let $R$ be a graded Noetherian ring and let $I \neq R$ be an ideal generated by $n$ homogeneous elements $a_{1}, \ldots, a_{n}$ in $R$. Let $P$ be a prime ideal minimal over I. Then h-ht $P \leq n$.

Proof. Replacing $R$ by $R_{S \backslash P}$, we may assume that $R$ is a graded Noetherian ring with $P$ as its unique maximal homogeneous ideal.

Suppose that there exists a chain $P=P_{0} \supset P_{1} \supset \cdots \supset P_{n+1}$ of homogeneous prime ideals of length $n+1$. Replacing $P_{1}$ by the ideal which is maximal in the family $\left\{P^{\prime}\right\}$ of the homogeneous prime ideals such that $P_{1} \subseteq P^{\prime} \subsetneq P$, we can also assume that there is no homogeneous prime ideal properly between $P_{1}$ and $P$.

Since $P$ is minimal over $I$, we can not have $I \subseteq P_{1}$ and so may assume that $a_{1} \notin P_{1}$. It follows that $P$ is the unique prime ideal minimal over $P_{1}+\left(a_{1}\right)$ and hence that $P=\sqrt{P_{1}+\left(a_{1}\right)}$. Since $P$ is finitely generated, there exists an integer $t \geq 1$ such that $P^{t} \subseteq P_{1}+\left(a_{1}\right)$. Consequently, we can write $a_{i}^{t}=$ $b_{i}+c_{i} a_{1}$, where $b_{i}, c_{i}$ are homogeneous elements of $R$ and $b_{i} \in P_{1}, i=2, \ldots, n$. 
Let $J=\left(b_{2}, \ldots, b_{n}\right)$, then $J$ is a homogeneous ideal contained in $P_{1}$. Since h-ht $P_{1} \geq n$, by the induction hypothesis, $P_{1}$ is not minimal over $J$. Let $Q$ be a homogeneous prime ideal such that $J \subseteq Q \subsetneq P_{1}$. The ideal $Q+\left(a_{1}\right)$ contains some power of each of the $a_{i}$ 's, whence $P$ is a prime ideal minimal over $Q+\left(a_{1}\right)$. We now pass to the graded Noetherian ring $R / Q$. Then the image $P / Q$ of $P$ is minimal over the image of $\left(a_{1}\right)$, but there is a chain $P / Q \supsetneq P_{1} / Q \supsetneq(0)$ of homogeneous prime ideals. This contradicts Theorem 3.5.

\section{The Krull-Akizuki theorem}

Let $M$ be a torsion-free graded module over a graded integral domain $R$. Then any two subsets of homogeneous elements of $M$ which are maximally linearly independent over $R$ have the same cardinality, which is defined to be the $h$-rank of $M$.

Lemma 4.1. Let $R$ be a graded Noetherian domain with h-dim $R=1, K$ its homogeneous quotient field, and $M$ a torsion-free graded $R$-module of finite h-rank $r$. Then for any nonzero homogeneous element $a \in R, \operatorname{hl}(M / a M) \leq$ $r \cdot \mathrm{hl}(R / a R)$.

Proof. First assume that $M$ is finitely generated. Choose homogeneous elements $x_{1}, \ldots, x_{r} \in M$ which are linearly independent over $R$ and set $E=$ $R x_{1}+\cdots+R x_{r}$. Then for any $x \in M$, there exists a nonzero homogeneous element $t \in R$ such that $t x \in E$. If we set $C=M / E$, then $C$ is a finitely generated graded $R$-module and $t C=0$ for some nonzero homogeneous element $t \in R$. Since $R / t R$ is a graded Noetherian ring with h-dim $(R / t R)=0$ and $C$ is a finitely generated graded $R / t R$-module, hl $C<\infty$ by Lemma 3.3.

Let $a$ be a nonzero homogeneous element of $R$. Then the exact sequence of graded $R$-module homomorphisms

$$
E / a^{n} E \rightarrow M / a^{n} M \rightarrow C / a^{n} C \rightarrow 0
$$

gives $\mathrm{hl}\left(M / a^{n} M\right) \leq \mathrm{hl}\left(E / a^{n} E\right)+\mathrm{hl}\left(C / a^{n} C\right) \leq \mathrm{hl}\left(E / a^{n} E\right)+\mathrm{hl}(C)$ for all $n>0$.

Now $E$ and $M$ are both torsion-free graded $R$-modules, $a^{i} E / a^{i+1} E \cong E / a E$ as graded $R$-modules and similarly for $M$. Hence we have $n \cdot \mathrm{hl}(M / a M) \leq$ $n \cdot \mathrm{hl}(E / a E)+\mathrm{hl} C$ for all $n>0$. Since both $\mathrm{hl}(M / a M)$ and $\mathrm{hl}(E / a E)$ are finite (Lemma 3.3), the previous inequality implies $\mathrm{hl}(M / a M) \leq \mathrm{hl}(E / a E)$.

Since $E=R x_{1}+\cdots+R x_{r}$ with $x_{1}, \ldots, x_{r}$ linearly independent homogeneous elements over $R$, we have $\mathrm{hl}(E / a E)=r \cdot \mathrm{hl}(R / a R)$. This completes the proof in the case that $M$ is finitely generated.

If $M$ is not finitely generated, take a finitely generated graded submodule $\bar{N}=R \overline{y_{1}}+\cdots+R \bar{y}_{s}$ of $\bar{M}=M / a M$, where each $\bar{y}_{i}$ is a homogeneous element of $\bar{M}$. Choose a homogeneous inverse image $y_{i}$ in $M$ for each $\bar{y}_{i}$ and set $M_{1}=$ $R y_{1}+\cdots+R y_{s}$. Then since $\bar{N}=\left(M_{1}+a M\right) / a M \cong M_{1} /\left(M_{1} \cap a M\right)$ as graded $R$-modules, we get $\mathrm{hl}(\bar{N})=\mathrm{hl}\left(M_{1} /\left(M_{1} \cap a M\right)\right) \leq \mathrm{hl}\left(M_{1} / a M_{1}\right) \leq$ $r^{\prime} \cdot \mathrm{hl}(R / a R) \leq r \cdot \mathrm{hl}(R / a R)$, where $r^{\prime}$ is the h-rank of $M_{1}$. The right-hand 
side is independent of $\bar{N}$, so that $\mathrm{hl} \bar{M} \leq r \cdot \mathrm{hl}(R / a R)<\infty$ and $\bar{M}$ is in fact finitely generated.

Theorem 4.2 (cf. Krull-Akizuki Theorem [12, Theorem 11.7]). Let $R \subseteq A$ be graded integral domains with homogeneous quotient fields $K \subseteq L$, respectively. Assume that $R$ is graded Noetherian with $\mathrm{h}-\operatorname{dim} R=1$ and $L$ is finite over $K$. Then $A$ is graded Noetherian with $\mathrm{h}-\operatorname{dim} A \leq 1$, and if $J$ is a nonzero homogeneous ideal of $A$, then $A / J$ is a graded $R$-module of finite h-length.

Proof. Since $K$ is a graded integral domain with h-dim $K=0$ and $L$ is a finitely generated graded $K$-module, $L$ has finite h-length by Lemma 3.2. Let $r$ be the h-length of $L$ as a graded $K$-module. Then $A$ is a torsion-free graded $R$-module of h-rank $r$. By Lemma 4.1, for any nonzero homogeneous element $c$ of $R$, the h-length of $A / c A$ as a graded $R$-module, $\mathrm{hl}_{R}(A / c A)$, is finite.

Now let $J$ be a nonzero homogeneous ideal of $A$ and $a$ a nonzero homogeneous element of $J$. Since $L$ is finite over $K$, the quotient field of $A$ is algebraic over the quotient field of $R$. Therefore, $a$ satisfies an equation of the form $c_{m} a^{m}+c_{m-1} a^{m-1}+\cdots+c_{1} a+c_{0}=0$, where the $c_{i}$ are homogeneous elements of $R$, not all zero. Since $A$ is an integral domain, we may assume that $c_{0} \neq 0$. Then $c_{0} \in J \cap R \backslash\{0\}$ and $\operatorname{so~hl}_{R}(A / J) \leq \mathrm{hl}_{R}\left(A / c_{0} A\right)<\infty$.

Moreover, since $\mathrm{hl}_{A}\left(J / c_{0} A\right) \leq \mathrm{hl}_{R}\left(J / c_{0} A\right) \leq \mathrm{hl}_{R}\left(A / c_{0} A\right)<\infty, J / c_{0} A$ is a finitely generated $A$-module and hence $J$ is a finitely generated $A$-module. Therefore, $A$ is a graded Noetherian ring.

If $P$ is a nonzero homogeneous prime ideal of $A$, then $A / P$ is a graded Noetherian domain of finite h-length as a graded $A$-module (and hence as a graded $A / P$-module). We will show that $P$ is a maximal homogeneous ideal of $A$. Let $I$ be a nonzero minimal homogeneous ideal of $A / P$ and let $x$ be a nonzero homogeneous element of $I$. Then $x I \subseteq I$. By the minimality of $I$, we have $x I=I$, and consequently, $x=x a$ for some $a \in I$. Then $a=1$, so $I=A / P$. Thus $A / P$ has no nontrivial homogeneous ideals and hence $P$ is a maximal homogeneous ideal of $A$. Therefore, $\mathrm{h}-\operatorname{dim} A \leq 1$.

\section{The Mori-Nagata theorem}

It was shown in [16, Section 2] and [15, Theorem 9.1] that the integral closure of a graded Noetherian domain is a graded Krull domain, which is a graded version of the Mori-Nagata theorem. The proof could be simplified by using induction as in $[2,10]$. The key will be Theorem 3.6 , which is a variation of the generalized principal ideal theorem.

We recall first the $v$ - and $t$-operations. For each nonzero fractional ideal $I$ of an integral domain $R$ with quotient field $F, I_{v}=\left(I^{-1}\right)^{-1}$, where $I^{-1}=$ $\{x \in F \mid x I \subseteq R\}$, and $I_{t}=\bigcup\left\{J_{v} \mid J\right.$ is a finitely generated subideal of $\left.I\right\}$. If $I=I_{v}$, then $I$ is called a $v$-ideal or a divisorial ideal, and if $I=I_{t}$, then $I$ is called a $t$-ideal.

We denote by h-t-Max $(R)$ the set of ideals of a graded integral domain $R$ which are maximal in the set of all proper homogeneous $t$-ideals of $R$. It 
is easy to check that any prime ideal minimal over a homogeneous $t$-ideal is a homogeneous $t$-ideal and any proper homogeneous $t$-ideal is contained in a maximal homogeneous $t$-ideal. Thus each ideal in the set h-t-Max $(R)$ is a prime ideal and the set h-t-Max $(R)$ is nonempty unless $R$ is equal to its homogeneous quotient field.

Lemma 5.1. Let $R$ be a graded integral domain and let $S$ be the set of nonzero homogeneous elements of $R$. Then $R=\bigcap_{P \in \mathrm{h}-\mathrm{t}-\operatorname{Max}(\mathrm{R})} R_{S \backslash P}$.

Proof. Since the intersection $\bigcap_{P \in \mathrm{h}-\mathrm{t}-\operatorname{Max}(\mathrm{R})} R_{S \backslash P}$ is a homogeneous overring of $R$, it suffices to show that each homogeneous element of the ring

$$
\bigcap_{P \in \mathrm{h}-\mathrm{t}-\mathrm{Max}(\mathrm{R})} R_{S \backslash P}
$$

is contained in $R$. Let $x$ be a homogeneous element of the ring

$$
\bigcap_{P \in \mathrm{h}-\mathrm{t}-\operatorname{Max}(\mathrm{R})} R_{S \backslash P}
$$

Then the ideal $\left(R:_{R} x\right)$ is a homogeneous $t$-ideal of $R$ which is not contained in any maximal homogeneous $t$-ideal. Therefore, we have $\left(R:_{R} x\right)=R$, which implies that $x \in R$.

Lemma 5.2. Let $R$ be a graded Noetherian domain and let $S$ be the set of nonzero homogeneous elements of $R$. Then the intersection

$$
R=\bigcap_{P \in \mathrm{h}-\mathrm{t}-\operatorname{Max}(\mathrm{R})} R_{S \backslash P}
$$

is homogeneously locally finite.

Proof. Let $a$ be a nonzero homogeneous element of $R$. Suppose that $a$ is contained in infinitely many maximal homogeneous $t$-ideals of $R$, say $P_{1}, P_{2}, \ldots$ Set $I_{k}=\bigcap_{i=1}^{k} P_{i}, k=1,2, \ldots$. Since each $P_{i}$ is a finitely generated $t$-ideal, it is divisorial. Therefore, $\left\{I_{k}\right\}_{k=1}^{\infty}$ is a strictly descending chain of homogeneous divisorial ideals. Thus we have an infinite chain of homogeneous integral ideals $a I_{1}^{-1} \subsetneq a I_{2}^{-1} \subsetneq \cdots$, which contradicts the fact that $R$ is a graded Noetherian domain.

Theorem 5.3 (cf. Mori-Nagata [13, Theorem 33.10]). Let $R$ be a graded Noetherian domain. Then the integral closure $R^{\prime}$ of $R$ is a graded Krull domain.

Proof. By Lemma 5.2, $R=\bigcap_{M \in \text { h-t-Max(R) }} R_{S \backslash M}$ is homogeneously locally finite. Then by [16, Lemma 2.2 and Lemma 2.3], we have

$$
R^{\prime}=\bigcap_{M \in \mathrm{h}-\mathrm{t}-\operatorname{Max}(\mathrm{R})}\left(R_{S \backslash M}\right)^{\prime}
$$

and it is also homogeneously locally finite. In view of [1, Theorem 5.15], it suffices to show that each $\left(R_{S \backslash M}\right)^{\prime}$ is a graded Krull domain. Thus by replacing 
$R$ with $R_{S \backslash M}$, we may assume that $R$ is a graded Noetherian domain with the unique maximal homogeneous ideal $M$. Then by Theorem 3.6, h-dim $R=$ h-ht $M<\infty$. We will prove the theorem by induction on h-dim $R:=n$.

If $n=1$, then $R^{\prime}$ is a completely integrally closed graded Noetherian domain (Theorem 4.2) and hence a graded Krull domain.

Let $n>1$. Assume that the assertion is true for the graded Noetherian domains with h-dimension less than $n$.

Let $A=R^{\prime} \cap R^{h g}$. Then $A$ is a homogeneous overring of $R$ contained in $R^{h g}$ and hence $A$ is a graded Noetherian domain by [16, Theorem 1.4]. Again by Lemma 5.2 and [16, Lemma 2.2 and Lemma 2.3], $A^{\prime}=\bigcap_{P \in \mathrm{h} \text {-t-Max(A) }}\left(A_{T \backslash P}\right)^{\prime}$ is homogeneously locally finite, where $T$ is the set of nonzero homogeneous elements of $A$. We will show that for each $P \in \mathrm{h}$-t-Max $(A),\left(A_{T \backslash P}\right)^{\prime}$ is a graded Krull domain.

Consider the case where $P$ is not a maximal homogeneous ideal of $A$. Since $A$ is integral over $R, P \cap R$ is not a maximal homogeneous ideal of $R$ and hence h-dim $A_{T \backslash P}=$ h-ht $P \leq$ h-ht $(P \cap R)<$ h-ht $M=$ h-dim $R=n$. By the induction hypothesis, $\left(A_{T \backslash P}\right)^{\prime}$ is a graded Krull domain.

Assume now that $P$ is a maximal homogeneous ideal of $A$. Then $P P^{-1}=P$ or $A$. Suppose that $P P^{-1}=P$. Then $P^{-1}=(P: P) \subseteq A^{\prime}=R^{\prime}$ since $P$ is a finitely generated ideal of $A$. Also, $P^{-1}=(A: P) \subseteq\left(R^{h g}: P \cap R\right)=\left(R^{h g}\right.$ : $M)=R^{h g}$. Therefore, $P^{-1} \subseteq R^{\prime} \cap R^{h g}=A$, which implies that $P_{v}=A$. But since $P$ is a finitely generated $t$-ideal of $A$, it is a divisorial ideal of $A$. We reach a contradiction. Therefore, $P$ is invertible.

We claim that h-ht $P=1$. Suppose that there exists a nonzero homogeneous prime ideal $Q$ properly contained in $P$. Then $Q \subseteq \bigcap_{k=1}^{\infty} P^{k}[4$, Theorem 7.6]. Choose a nonzero homogeneous element $a \in Q$. Then we have an infinite chain of homogeneous integral ideals $a P^{-1} \subsetneq a\left(P^{2}\right)^{-1} \subsetneq \cdots$, which contradicts the fact that $A$ is a graded Noetherian domain.

Thus we have h-dim $A_{T \backslash P}=$ h-ht $P=1$. Then by Theorem $4.2,\left(A_{T \backslash P}\right)^{\prime}$ is a graded Krull domain.

Since $A^{\prime}=\bigcap_{P \in \mathrm{h} \text {-t-Max(A) }}\left(A_{T \backslash P}\right)^{\prime}$ is homogeneously locally finite and each $\left(A_{T \backslash P}\right)^{\prime}$ is a graded Krull domain, it follows that $A^{\prime}=R^{\prime}$ is a graded Krull domain.

\section{The Nagata theorem}

In this section we generalize the following Nagata theorem to a graded Noetherian domain: If $R$ is a Noetherian domain with $\operatorname{dim} R \leq 2$, then the integral closure $R^{\prime}$ of $R$ is a Noetherian domain. We follow the proof by Nishimura [14]. As in his proof, the graded versions of the Krull-Akizuki theorem (Theorem 4.2), the Mori-Nagata theorem (Theorem 5.3), and the Mori-Nishimura theorem (Theorem 6.7) will play critical roles in our proof of the main theorem, Theorem 6.8. 
Lemma 6.1. Let $M$ be a finitely generated graded module over a graded Noetherian ring $R$. Then $M$ is a graded Noetherian $R$-module, i.e., every graded $R$-submodule of $M$ is finitely generated.

Proof. We prove the assertion by induction on the number of generators of $M$. If $M$ is generated by the empty set, then $M=\{0\}$ and the assertion is obvious. Assume that $M=R x_{1}+\cdots+R x_{n}$, where each $x_{i}$ is a homogeneous element of $M$. Set $M^{\prime}=R x_{2}+\cdots+R x_{n}$. Then by the induction hypothesis, $M^{\prime}$ is graded Noetherian.

Now let $N$ be a graded $R$-submodule of $M$. Let $I$ be the set of elements $a$ of $R$ such that $a x_{1} \in N+M^{\prime}$, i.e., $I=\left(\left(N+M^{\prime}\right):_{R} x_{1}\right)$. Then since $I$ is a homogeneous ideal of $R, I$ is generated by a finite number of homogeneous elements of $R$, say $a_{1}, \ldots, a_{m}$. For each $i=1, \ldots, m$, let $y_{i}$ be a homogeneous element of $N$ such that $y_{i}-a_{i} x_{1} \in M^{\prime}$, and let $N^{\prime}$ be the graded $R$-submodule $R y_{1}+\cdots+R y_{m}$ of $N$. Then $N=N^{\prime}+\left(N \cap M^{\prime}\right)$. Since $M^{\prime}$ is graded Noetherian, $N \cap M^{\prime}$ is finitely generated and hence $N$ is finitely generated.

Therefore, $M$ is graded Noetherian.

Lemma 6.2. Let $R \subset A$ be graded rings with $A$ integral over $R$. Then the $h$-dimension of $A$ equals the $h$-dimension of $R$. In particular, if $R$ is a graded Noetherian domain, then h-dim $R^{\prime}=\mathrm{h}-\operatorname{dim} R$.

Proof. Consider a chain $P_{1} \subset P_{2} \subset \cdots \subset P_{n}$ of homogeneous prime ideals of $R$. Then by GU, there exists a chain $Q_{1} \subset Q_{2} \subset \cdots \subset Q_{n}$ of prime ideals of $A$ such that $Q_{i} \cap R=P_{i}$. Let $Q_{i}^{*}$ be the ideal generated by the homogeneous elements in $Q_{i}$. Then $Q_{i}^{*}$ is a homogeneous prime ideal of $A$ such that $Q_{i}^{*} \cap R=P_{i}$. By INC, $Q_{i}^{*}=Q_{i}$, i.e., $Q_{i}$ is homogeneous. Therefore, h-dim $R \leq \mathrm{h}-\operatorname{dim} A$.

Conversely, let $Q_{1} \subset \cdots \subset Q_{n}$ be a chain of distinct homogeneous prime ideals of $A$. Then by INC, $Q_{1} \cap R \subset Q_{2} \cap R \subset \cdots \subset Q_{n} \cap R$ is a chain of distinct prime ideals of $R$. Since each $Q_{i} \cap R$ is a homogeneous ideal of $R$, h-dim $A \leq \mathrm{h}-\operatorname{dim} R$.

Lemma 6.3. Let $R$ be a graded ring, and let $I_{1}, \ldots, I_{n}$ be homogeneous ideals in $R$ such that $\bigcap_{i=1}^{n} I_{i}=(0)$ and each $R / I_{i}$ is graded Noetherian. Then $R$ is graded Noetherian.

Proof. It suffices to consider the case $n=2$.

Since $\left(I_{1}+I_{2}\right) / I_{2}$ is a homogeneous ideal of the graded Noetherian ring $R / I_{2}$, there exist a finite number of homogeneous elements of $I_{1}$, say $a_{1}, \ldots, a_{r}$, such that $\left(I_{1}+I_{2}\right) / I_{2}=\left(\left(a_{1}, \ldots, a_{r}\right)+I_{2}\right) / I_{2}$, i.e., $I_{1}+I_{2}=\left(a_{1}, \ldots, a_{r}\right)+I_{2}$. By assumption that $I_{1} \cap I_{2}=(0)$, we have $I_{1}=\left(a_{1}, \ldots, a_{r}\right)$. Similarly, $I_{2}$ is finitely generated.

Now let $P$ be a homogeneous prime ideal of $R$. Then $I_{1} \cap I_{2}=(0) \subseteq P$, and hence $I_{1} \subseteq P$ or $I_{2} \subseteq P$. Assume that $I_{1} \subseteq P$. Then since $I_{1}$ and $P / I_{1}$ are finitely generated, so is $P$. Therefore, $R$ is a graded Noetherian ring. 
Lemma 6.4. Let $R$ be a graded ring and let $M$ be a faithful graded $R$-module. If $M$ is a graded Noetherian $R$-module, then $R$ is a graded Noetherian ring.

Proof. Since $M$ is a graded Noetherian $R$-module, it is finitely generated. Write $M=R x_{1}+\cdots+R x_{n}$, where each $x_{i}$ is a homogeneous element of $M$. Then each $R x_{i}$ is a graded Noetherian $R$-module and $R x_{i} \cong R /\left(0:_{R} x_{i}\right)$ as graded $R$-modules. Hence $R /\left(0:_{R} x_{i}\right)$ is a graded Noetherian ring. Now $\bigcap_{i=1}^{n}\left(0:_{R}\right.$ $\left.x_{i}\right)=\left(0:_{R} M\right)=(0)$, because $M$ is a faithful $R$-module. Therefore, the conclusion follows from Lemma 6.3.

Lemma 6.5. Let $R$ be a graded ring and let $M$ be a graded $R$-module which is finitely generated and faithful over $R$. Assume that the set of submodules of $M$ of the form IM with $I$ a homogeneous ideal of $R$ satisfies the ascending chain condition. Then $R$ is a graded Noetherian ring.

Proof. By Lemma 6.4, it suffices to show that $M$ is a graded Noetherian $R$ module. Suppose not. Let $\mathcal{S}$ be the set of submodules of $M$ of the form $I M$, where $I$ is a homogeneous ideal of $R$ and $M / I M$ is not a graded Noetherian $R$-module. Since $(0) \in \mathcal{S}, \mathcal{S}$ is non-empty. By assumption, $\mathcal{S}$ has a maximal element, say $I M$.

Replacing $M$ by $M / I M$ and $R$ by $R /\left(0:_{R} M / I M\right)$, we may assume that $M$ is not a graded Noetherian $R$-module, but for any nonzero homogeneous ideal $I$ of $R, M / I M$ is a graded Noetherian $R$-module.

Let $\mathcal{T}$ be the set of graded $R$-submodules $N$ of $M$ such that $M / N$ is a faithful $R$-module. Since $\{0\} \in \mathcal{T}, \mathcal{T}$ is non-empty. Give a partial ordering on $\mathcal{T}$ by set inclusion.

We will show that $\mathcal{T}$ is inductive; that is, let $\left\{N_{\lambda}\right\}_{\lambda \in \Lambda}$ be a chain in $\mathcal{T}$, then $N:=\bigcup_{\lambda \in \Lambda} N_{\lambda}$ is in $\mathcal{T}$. It is obvious that $N$ is a graded $R$-submodule of $M$. Suppose that $M / N$ is not faithful over $R$. Then there exists a nonzero element $a \in R$ such that $a(M / N)=0$, i.e., $a M \subseteq N$. Since $M$ is finitely generated over $R, a M \subseteq N_{\lambda}$, i.e., $a\left(M / N_{\lambda}\right)=0$ for some $\lambda \in \Lambda$. This contradicts the fact that $M / N_{\lambda}$ is faithful over $R$.

By Zorn's lemma, $\mathcal{T}$ has a maximal element, say $N_{0}$. Then $M / N_{0}$ is a faithful graded $R$-module. If $M / N_{0}$ is a graded Noetherian $R$-module, then $R$ is a graded Noetherian ring by Lemma 6.4 , and hence $M$ is a graded Noetherian $R$-module by Lemma 6.1 . This contradicts our hypothesis.

Replacing $M$ by $M / N_{0}$, we arrive at a graded $R$-module $M$ with the following properties:

(1) $M$ is not a graded Noetherian $R$-module;

(2) for any nonzero homogeneous ideal $I$ of $R, M / I M$ is a graded Noetherian $R$-module;

(3) for any nonzero graded submodule $N$ of $M, M / N$ is not faithful over $R$.

Now let $N$ be any nonzero graded submodule of $M$. By $(3),\left(0:_{R} M / N\right) \neq$ (0). Since $\left(0:_{R} M / N\right)$ is a nonzero homogeneous ideal of $R$, there exists a 
nonzero homogeneous element $a \in R$ such that $a(M / N)=0$, i.e., $a M \subseteq N$. By (2), $M / a M$ is a graded Noetherian $R$-module, so that $N / a M$ is finitely generated. But since $M$ is finitely generated, so is $a M$, and hence $N$ is finitely generated. Thus, $M$ is a graded Noetherian $R$-module, which contradicts (1).

Theorem 6.6 (cf. Eakin-Nagata [12, Theorem 3.7]). Let $A$ be a graded Noetherian ring and let $R$ be a graded subring of $A$ such that $A$ is finite over $R$. Then $R$ is also a graded Noetherian ring.

Proof. Since $A$ is an extension ring of $R$, it is a faithful $R$-module. Therefore, the conclusion follows directly from Lemma 6.5.

Theorem 6.7 (cf. Mori-Nishimura [12, Theorem 12.7]). Let $R$ be a graded Krull domain and let $X_{H}(R)$ be the set of homogeneous prime ideals of $R$ of height 1. If $R / P$ is a graded Noetherian domain for every $P \in X_{H}(R)$, then $R$ is a graded Noetherian domain.

Proof. By [18, Lemma 2.3], it suffices to show that each nonzero homogeneous prime ideal $Q$ of $R$ is finitely generated. Choose a nonzero homogeneous element $a \in Q$. Then by [1, Proposition 5.6], $a R=P_{1}^{\left(n_{1}\right)} \cap \cdots \cap P_{r}^{\left(n_{r}\right)}$, where $P_{1}, \ldots, P_{r}$ are distinct prime ideals in $X_{H}(R)$ and $n_{1}, \ldots, n_{r}$ are positive integers. If each $R / P_{i}^{\left(n_{i}\right)}$ is graded Noetherian, then by Lemma $6.3, R / a R$ is graded Noetherian, and hence $Q / a R$ is finitely generated. Thus it follows that $Q$ is finitely generated.

Therefore, it is enough to show that $R / P^{(n)}$ is graded Noetherian for every $P \in X_{H}(R)$ and positive integer $n$.

Choose a nonzero homogeneous element $a \in P \backslash P^{(2)}$. Then $a R=P \cap$ $P_{2}^{\left(n_{2}\right)} \cap \cdots \cap P_{r}^{\left(n_{r}\right)}$, where $P, P_{2}, \ldots, P_{r}$ are distinct prime ideals in $X_{H}(R)$ and $n_{2}, \ldots, n_{r}$ are positive integers. Choose a homogeneous element $b_{i} \in P_{i} \backslash P$

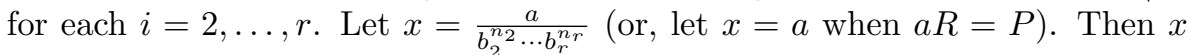
is a homogeneous element in $R_{S}$, where $S$ is the set of nonzero homogeneous elements of $R$. By [1, Proposition 5.5], $R_{q}$ is a DVR for each $q \in X_{H}(R)$. Let $v_{q}$ be the normalized additive valuation determined by $R_{q}$. Then $v_{P}(x)=1$ and $v_{q}(x) \leq 0$ for all $q \in X_{H}(R) \backslash\{P\}$.

Set $A=R[x]$, then $A$ is a homogeneous overring of $R$.

Claim: $A / x A \cong R / P$ as graded rings.

Since $v_{P}(x)=1, x A \subseteq x R_{P}=P R_{P}$ and hence $x A \cap R \subseteq P$. Conversely, let $y$ be a homogeneous element in $P$. Then since $v_{P}\left(\frac{y}{x}\right) \geq 0$ and $v_{q}\left(\frac{y}{x}\right) \geq 0$ for all $q \in X_{H}(R) \backslash\{P\}, \frac{y}{x} \in R_{S} \cap\left(\bigcap_{q \in X_{H}(R)} R_{q}\right)=R$ [1, Theorem 5.8], and so $y \in x R \subseteq x A$. Therefore, $P \subseteq x A \cap R$. Thus we have $P=x A \cap R$. Moreover, $A=R+x A$, and hence $A / x A \cong R /(x A \cap R)=R / P$. 
By assumption, $A / x A$ is a graded Noetherian ring. Since any homogeneous prime ideal of $A$ containing $x^{n} A$ contains $x A, A / x^{n} A$ is also a graded Noetherian ring by $\left[18\right.$, Lemma 2.3]. Note that $A / x^{n} A$ is a graded $R /\left(x^{n} A \cap R\right)$ module generated by the images $1, x, \ldots, x^{n-1}$. Then it follows from Theorem 6.6 that $R /\left(x^{n} A \cap R\right)$ is a graded Noetherian ring. Moreover, since $x^{n} A \cap R \subseteq x^{n} R_{P} \cap R=P^{n} R_{P} \cap R=P^{(n)}, R / P^{(n)}$ is also a graded Noetherian ring.

Theorem 6.8 (cf. Nagata Theorem [14]). Let $R$ be a graded Noetherian domain with h-dim $R \leq 2$. Then the integral closure $R^{\prime}$ is also a graded Noetherian domain with h-dim $R^{\prime} \leq 2$.

Proof. By Theorem 4.2 and [16, Lemma 2.2 and Lemma 2.3], we may assume that h-dim $R=2$. By Theorem 5.3 and Lemma $6.2, R^{\prime}$ is a graded Krull domain with h-dim $R^{\prime}=2$. Therefore, by Theorem 6.7 , it suffices to show that $R^{\prime} / P^{\prime}$ is graded Noetherian for every $P^{\prime} \in X_{H}\left(R^{\prime}\right)$.

If $P^{\prime}$ is a maximal homogeneous ideal of $R^{\prime}$, then $R^{\prime} / P^{\prime}$ has no nontrivial homogeneous ideals and hence it is trivially graded Noetherian.

Assume that $P^{\prime}$ is not a maximal homogeneous ideal of $R^{\prime}$. Set $P=P^{\prime} \cap R$. Then since $R^{\prime}$ is integral over $R, P$ is not a maximal homogeneous ideal of $R$. Since h-dim $R=2$, h-ht $P=1$ and each (homogeneous) prime ideal of $R^{\prime}$ lying over $P$ is of h-height 1 .

Since $R^{\prime}$ is a graded Krull domain, there exist only a finite number of homogeneous prime ideals of $R^{\prime}$ lying over $P$, say $P^{\prime}, P_{2}^{\prime}, \ldots, P_{r}^{\prime}$. Choose a homogeneous element $x_{i} \in P^{\prime} \backslash P_{i}^{\prime}$ for each $i=2, \ldots, r$. Since $R\left[x_{2}, \ldots, x_{r}\right]$ is a graded subring of $R^{\prime}$ and it is finite over $R, R\left[x_{2}, \ldots, x_{r}\right]$ is a graded Noetherian domain with h-dimension 2 by Lemma 6.1 and Lemma 6.2.

Replacing $R$ by $R\left[x_{2}, \ldots, x_{r}\right]$, we may assume that $P^{\prime}$ is the unique homogeneous prime ideal of $R^{\prime}$ lying over $P$.

Since $R_{S \backslash P}$ is a graded Noetherian domain with h-dim $R_{S \backslash P}=1$, it follows from Theorem 4.2 that $\left(R_{S \backslash P}\right)^{\prime} / a\left(R_{S \backslash P}\right)^{\prime}=R_{S \backslash P}^{\prime} / a R_{S \backslash P}^{\prime}$ is a finite $R_{S \backslash P}$-module for each nonzero homogeneous element $a$ of $R_{S \backslash P}$. In particular, $R_{S \backslash P}^{\prime} / P^{\prime} R_{S \backslash P}^{\prime}$ is a finite $R_{S \backslash P} / P R_{S \backslash P}$-module. Note that $R_{S \backslash P}^{\prime} / P^{\prime} R_{S \backslash P}^{\prime}$, $R_{S \backslash P} / P R_{S \backslash P}$ are homogeneous quotient fields of $R^{\prime} / P^{\prime}, R / P$, respectively. Since $R / P$ is a graded Noetherian domain with h- $\operatorname{dim} R / P=1, R^{\prime} / P^{\prime}$ is graded Noetherian by Theorem 4.2 again.

\section{Concluding remarks}

We have only investigated the results which can be generalized to graded rings. But there are some important properties of rings whose graded versions do not hold. We end this paper by mentioning two well-known negative results.

One is among the most useful tools in the theory of commutative rings: If an ideal $I$ is not contained in any of the prime ideals $P_{1}, \ldots, P_{n}$, then there exists an element $a \in I \backslash \bigcup_{i=1}^{n} P_{i}$. However, the corresponding homogeneous 
version does not hold. That is, for a homogeneous ideal $I$ and homogeneous prime ideals $P_{1}, \ldots, P_{n}$, it may happen that all homogeneous elements in $I$ are in $\bigcup_{i=1}^{n} P_{i}$ even though $I$ is not contained in any of $P_{i}$.

Example 7.1. Let $R=k[X, Y]=\bigoplus_{(n, m) \in \mathbb{N}_{0} \times \mathbb{N}_{0}} k X^{n} Y^{m}$. Then h-Spec $(R)=$ $\{(0),(X),(Y),(X, Y)\}$. Note that $(X, Y) \nsubseteq(X) \cup(Y)$, but there does not exist any homogeneous element in $(X, Y) \backslash(X) \cup(Y)$.

The other is that if $P \subset Q$ are prime ideals in a Noetherian ring such that ht $(Q / P)=2$, then there are infinitely many prime ideals between $P$ and $Q$. Ratliff Jr. and Rush show that the analogous result concerning homogeneous prime ideals in a $\mathbb{Z}$-graded Noetherian ring fails to hold. For specific examples, see [17, Remark 3.12 and Example 4.9].

\section{References}

[1] D. D. Anderson and D. F. Anderson, Divisibility properties of graded domains, Canad. J. Math. 34 (1982), no. 1, 196-215.

[2] A. Bouvier and G. Germain, A concise proof of a theorem of Mori-Nagata, C. R. Math. Rep. Acad. Sci. Canada 1 (1978/79), no. 6, 315-318.

[3] L. Budach, Struktur Noetherscher kommutativer Halbgruppen, Monatsb. Deutsch. Akad. Wiss. Berlin 6 (1964), 85-88.

[4] R. Gilmer, Multiplicative Ideal Theory, Dekker, New York, 1972.

[5] S. Goto and K. Watanabe, On graded rings. I, J. Math. Soc. Japan 30 (1978), no. 2, 179-213.

[6] - On graded rings. II. ( $\mathbb{Z}^{n}$-graded rings), Tokyo J. Math. 1 (1978), no. 2, 237-261.

[7] S. Goto and K. Yamagishi, Finite generation of Noetherian graded rings, Proc. Amer. Math. Soc. 89 (1983), no. 1, 41-44.

[8] W. Heinzer, On Krull overrings of a Noetherian domain, Proc. Amer. Math. Soc. 22 (1969), 217-222.

[9] W. Heinzer and M. Roitman, The homogeneous spectrum of a graded commutative ring, Proc. Amer. Math. Soc. 130 (2002), no. 6, 1573-1580.

[10] B. G. Kang, Integral closure of rings with zero divisors, J. Algebra 162 (1993), no. 2, 317-323.

[11] I. Kaplansky, Commutative Rings, Allyn and Bacon, Boston, 1970.

[12] H. Matsumura, Commutative Ring Theory, Cambridge Univ. Press, Cambridge, 1986.

[13] M. Nagata, Local Rings, Interscience, New York, 1962.

[14] J. Nishimura, Note on integral closures of a Noetherian integral domain, J. Math. Kyoto Univ. 16 (1976), no. 1, 117-122.

[15] J. S. Okon, D. E. Rush, and L. J. Wallace, A Mori-Nagata theorem for lattices and graded rings, Houston J. Math. 31 (2005), no. 4, 973-997.

[16] M. H. Park, Integral closure of graded integral domains, Comm. Algebra 35 (2007), no. 12, 3965-3978.

[17] L. J. Ratliff Jr. and D. E. Rush, Two notes on homogeneous prime ideals in graded Noetherian rings, J. Algebra 264 (2003), no. 1, 211-230.

[18] D. E. Rush, Noetherian properties in monoid rings, J. Pure Appl. Algebra 185 (2003), no. 1-3, 259-278.

[19] O. Zariski and P. Samuel, Commutative Algebra. Vol. I, Graduate Texts in Mathematics, Vol. 28. Springer-Verlag, New York-Heidelberg-Berlin, 1975. 
Chang Hwan Park

Department of Mathematics

Chung-Ang University

SeOul 156-756, KoreA

E-mail address: mars_chang@naver.com

Mi Hee Park

Department of Mathematics

Chung-Ang University

SEOUl 156-756, KoreA

E-mail address: mhpark@cau.ac.kr 